Palabra Clave (La Plata), abril 2017, vol. 6, n² 2, e019. ISSN 1853-9912

Universidad Nacional de La Plata.

Facultad de Humanidades y Ciencias de la Educación.

Departamento de Bibliotecología

\title{
Implementación de las RDA en la Universidad Nacional Autónoma de México
}

\author{
RDA Implementation at the National Autonomous University of \\ Mexico
}

\author{
Filiberto Felipe Martínez Arellano *, Evelia Santana Chavarría *, \\ Patricia de la Rosa Valgañón $* *$
}

* Universidad Nacional Autónoma de México. Instituto de Investigaciones Bibliotecológicas y de la Información, ** Universidad Nacional Autónoma de México. Dirección General de Bibliotecas, México | felipe@unam.mx, santanae@biblional.bibliog.unam.mx, pattyrov@unam.mx

\section{PALABRAS CLAVE RESUMEN}

Estándares de catalogación

La Universidad Nacional Autónoma de México (UNAM) es la más grande e importante institución educativa de México y de América Latina. Atiende a más de 340.000 alumnos de posgrado, licenciatura y bachillerato. Ofrece 41 programas de posgrado y 118 carreras de licenciatura. Asimismo, la Biblioteca Nacional de México (BNM) se encuentra dentro de la estructura de UNAM, siendo la encargada de salvaguardar y preservar la memoria bibliográfica de México, la cual se encuentra conformada por más de 1.250 .000 libros y documentos que se encuentran resguardados en la BNM. Por otro lado, el Sistema Bibliotecario y de Información de la UNAM (SIBIUNAM) está integrado por 135 bibliotecas con un acervo de aproximadamente 1.700 .000 títulos de libros y 6.000 .000 volúmenes, siendo coordinado por la Dirección General de Bibliotecas. Adicionalmente, dentro de la organización de la UNAM se encuentra el Instituto de Investigaciones Bibliotecológicas y de la Información (IIBI), en donde se llevan a cabo proyectos de investigación teórica y aplicada en diversas áreas de investigación, siendo una de ellas la organización de la información y, dentro de ésta, se abordan los estándares de catalogación. En el presente artículo se tratan los retos que la adopción del nuevo estándar de catalogación RDA ha significado para estas tres entidades de la UNAM, así como las acciones desarrolladas en cada una de ellas. Se comparten las experiencias de la Biblioteca Nacional de México (BNM) sobre la implementación de RDA. Se presentan algunas situaciones problemáticas, así como la solución de éstas. Se incluye la participación de la BNM en foros, para comunicar los trabajos que se realizaban sobre la catalogación con este código. También se aborda la actualización y capacitación del personal, la participación en los programas de catalogación cooperativa y el análisis de las RDA en equipos de trabajo, así como los acuerdos para su implementación. Se hace mención a los cambios en los flujos de trabajo, los apoyos tecnológicos que se necesitaron, la situación actual y los retos futuros de la implementación de RDA. Asimismo, se presentan el análisis y los procesos para la aplicación de RDA en los materiales adquiridos por el Sistema Bibliotecario y de Información de la UNAM (SIBIUNAM). Se presentan también los retos que se enfrentaron durante este cambio. Adicionalmente, se describen diversos aspectos relativos a los registros existentes en catálogo LIBRUNAM, así como su cambio retrospectivo; también se aborda lo correspondiente a la generación de nuevos registros con el consiguiente agregado de etiquetas MARC, así como los retos que ha significado la aplicación de las RDA. Finalmente, se abordan algunas de las acciones que el Instituto de Investigaciones Bibliotecológicas ha liderado para la adopción de las RDA como la organización de los Encuentros de Catalogación y Metadatos, los cuales se han convertido en el foro para la discusión de los problemas relacionados con la adopción y aplicación de las RDA en las bibliotecas de la UNAM, así como en otras bibliotecas de México y de América Latina. Asimismo, las recomendaciones que han sido discutidas en estos eventos académicos para la formación dentro de las escuelas de bibliotecología, de profesionales capaces de utilizar las RDA.

\section{KEYWORDS ABSTRACT}

Cataloging standards

The Universidad Nacional Autónoma de México (UNAM) [National Autonomous University of Mexico] is the largest and most important educational institution in Mexico and Latin America. It serves more than 340,000 graduate, bachelor and high school students. It offers 41 graduate programs and 118 undergraduate ones. Moreover, the Biblioteca Nacional de México (BNM) [National Library of Mexico] is into the UNAM structure, playing the role of safeguarding and preserving the bibliographical memory of Mexico, which is integrated by more than $1,250,000$ books and documents that are sheltered into this library. Moreover, the Sistema Bibliotecario y de Información de la UNAM (SIBIUNAM) [UNAM Library and Information System] is composed of 135 libraries with a collection of approximately 1700.000 (STBles collection of approximately $1,70.000$ book titles and $6,000.000$ volumes, which is com General de Bibliotecas de la UNAM [UNAM General Directorate of Libraries]. Additionally, in the UNAM organization is placed the Instituto de Investigaciones Bibliotecologicas y de la Informacion (IIBI) [Institute of Research in Library and Information Sciences], where and within this one, cataloging standards are approached. This article addresses the challenges that the adoption of the new RDA and within this one, cataloging standards are approached. This article addresses the challenges that the adoption of the new RDA cataloging standard has represented for these three UNAM organizations, as well as the actions developed in each one of them. The Biblioteca Nacional México (BNM) experiences on RDA implementation are shared. Some problematic situations, as well as the solution for these ones, are presented. It is also included the BNM participation in forums, to communicate the work that was done on cataloging using the new code. It also is addressed the updating and training of staf, participation in cooperative cataloging programs and the RDA analysis in work teams, as well as the agreements for their implementation. Also, a mention is made about the changes in workflows, the technological support that was needed, the curef RDA in the Biblichar Bibliotecario y delation, 作 ches; the aticle also deas with the gen c la Informacion (IIBI) has carried out for the RDA a doption as the Cataloging and Metadata Meetings organization, Which have de la Informacion (IIBI) has carried out for the RDA adoption as the Cataloging and Metadata Meetings organization, which have become a forum for problem discussion related on RDA adoption and application at UNAM libraries, as well as in other Mexican and professional training in LIS schools to be able of using RDA.

Recibido: 15 de diciembre de 2016 | Aceptado: 28 de febrero de 2017 | Publicado: 20 de abril de 2017 


\section{Introducción}

La Universidad Nacional Autónoma de México (UNAM) es la universidad pública más grande de México y de América Latina. En su campus central y en otros distribuidos en la Ciudad de México y en diferentes lugares del país (León Guanajuato y Morelia Michoacán, entre otros), la UNAM atiende a una población de alrededor de 340.000 alumnos, de los cuales 28.000 cursan algunos de los 41 posgrados que ofrece, y 206.000 alguna de las 118 carreras profesionales a nivel licenciatura que se imparten en esta institución. Las actividades de investigación se realizan en 22 institutos, 8 centros y 5 programas del área científica y en 11 institutos, 6 centros y 6 programas del área de las Humanidades y las Ciencias Sociales. Su planta académica se encuentra conformada por alrededor de 39.500 profesores, investigadores y técnicos académicos, de los cuales 12.000 son de tiempo completo. Asimismo, cabe mencionar que el $30 \%$ de los artículos científicos publicados por académicos mexicanos es publicado por investigadores de la UNAM y que la institución publica en promedio 6 libros por día (UNAM, 2016). Dentro de su estructura, la UNAM cuenta con tres importantes instituciones bibliotecarias, la Biblioteca Nacional de México, la Dirección General de Bibliotecas y el Instituto de Investigaciones Bibliotecológicas.

La Biblioteca Nacional de México fue fundada el 30 de noviembre de 1867 por el entonces presidente Benito Juárez y desde 1914 existe una estrecha vinculación con la Universidad Nacional de México, la cual se formalizó en 1929 cuando ésta obtuvo su autonomía, siendo desde entonces parte integrante de la UNAM. La Biblioteca Nacional constituye el máximo repositorio bibliográfico del país: su acervo resguarda y conserva más de 1.250 .000 libros y documentos. Algunos aspectos importantes de la misión de la Biblioteca Nacional de México son "Normar las actividades bibliotecológicas y publicar la bibliografía nacional" y "Divulgar el contenido de sus colecciones mediante: catálogos, bibliografías, folletos, exposiciones, conferencias, páginas web, visitas guiadas y otros medios" (UNAM. Biblioteca Nacional de México, 2016). Asimismo, una de las funciones primordiales de la Biblioteca Nacional de México es la elaboración de registros bibliográficos de los materiales que ingresan mediante el depósito legal, compra y donación. El acervo bibliográfico de la Biblioteca Nacional de México se encuentra registrado el catálogo Nautilius (http://catalogo.iib.unam.mx/F/-/?func=find-b0\&local base=BNM) La Dirección General de Bibliotecas (DGB) es la dependencia encargada de la tarea de coordinar el Sistema Bibliotecario y de Información de la Universidad Nacional Autónoma de México, el cual se encuentra integrado por 135 bibliotecas y 33 colecciones localizadas en las distintas facultades, escuelas, institutos y centros de investigación de la UNAM. Por otro lado, su sistema Bibliotecario (SIBIUNAM) está integrado por 135 bibliotecas con un acervo de 1.700 .000 títulos de libros y de 6.000 .000 volúmenes. Los materiales adquiridos por las bibliotecas son procesados técnicamente por la DGB con el sistema de clasificación de la Biblioteca del Congreso de Estados Unidos (LC) y las normas RDA (Recursos, Descripción y Acceso). La riqueza documental de SIBIUNAM se puede localizar mediante los catálogos colectivos que reúnen los recursos existentes en todas las unidades que lo conforman. Uno de los principales catálogos colectivos de este sistema es LIBRUNAM, que contiene los registros catalográficos de los más de 1.700.000 libros existentes en las diferentes bibliotecas de SIBIUNAM (UNAM. Dirección General de Bibliotecas, 2013).

El Instituto de Investigaciones Bibliotecológicas y de la Información tiene su origen en el Centro Universitario de Investigaciones Bibliotecológicas y de la Información, el cual "fue establecido en la UNAM el 14 de diciembre de 1981 con la finalidad de llevar a cabo investigaciones teóricas y aplicadas sobre los fenómenos relacionados con la información 
registrada en impresos y otros medios: sus formas de generación, su selección y adquisición, su organización y representación. Asimismo, otra de sus atribuciones era investigar "sobre los problemas concernientes con los medios, procesos y tecnologías utilizados para el almacenamiento, recuperación y distribución de la información" (Martinez Arellano, 2007). Después de 30 años de trabajo y desarrollo académico, el CUIB se transformó en el actual Instituto de Investigaciones Bibliotecológicas y de la Información. Actualmente, la agenda de investigación del IIBI está integrada por nueve áreas: Organización de la Información y el Conocimiento; Tecnologías de la Información y el Conocimiento; Información, Conocimiento y Sociedad; Metría de la Información y del Conocimiento Científico; Usuarios de la Información; Lectura; Servicios y Recursos de Información; Educación Bibliotecológica e Historia y Fundamentos de la Bibliotecología y Estudios de la información. En el área de Organización de la Información y el Conocimiento, actualmente se desarrollan los siguientes proyectos de investigación: "Impacto de las RDA (Resource Description and Access) en los sistemas de recuperación de la información y "Estandarización de los elementos bibliográficos en el entorno de la descripción y acceso a los recursos" (UNAM. Instituto de Investigaciones Bibliotecológicas y de la Información, 2017).

Estas tres entidades de la UNAM se encuentran directamente involucradas con el área de la catalogación de recursos de información y se desarrollan en cada una de ellas proyectos y actividades relacionados con la aplicación del nuevo estándar de catalogación RDA. Dichas actividades son presentadas en la siguiente parte de este artículo.

\section{Implementación de RDA en la Biblioteca Nacional de México}

Como ha sido mencionado, una función primordial de la Biblioteca Nacional de México es la elaboración de registros bibliográficos de los materiales que ingresan mediante Depósito Legal, compra y donación.

El Departamento de Catalogación es el responsable de esta tarea. Como productos del proceso de catalogación descriptiva y temática de los materiales documentales, se cuenta con:

Catálogo electrónico de las distintas colecciones de la Biblioteca Nacional de México

Registros bibliográficos de las publicaciones editadas en México para la formación de la bibliografía nacional contemporánea (denominada Bibliografía Mexicana), función primordial de toda biblioteca nacional (UNAM. Biblioteca Nacional de México, 2016).

Los catálogos de la Biblioteca y Hemeroteca Nacionales de México pueden ser consultados en la siguiente dirección: http://catalogo.iib.unam.mx/otroscatbnm. Adicionalmente, el catálogo de la Bibliografía Mexicana es accesible en la dirección: http://catalogo.iib.unam.mx/F/-/? func $=$ login\&local base $=$ bibmex

La Biblioteca Nacional de México ha organizado la información de sus colecciones documentales aplicando técnicas y procedimientos de acuerdo a cada época, por lo que en referencia a la aplicación de estándares internacionales de control bibliográfico se ha documentado debidamente para poder implementarlos y estar a la par de otros órganos bibliográficos nacionales. En este sentido, la aplicación del código RDA (Descripción y Acceso a los Recursos) ha transitado por un proceso continuo para ser implementada. En dicho proceso se ha tenido cuidado de que en las etapas de su implementación se formaran equipos de trabajo con las 
instituciones que lo analizaban desde la teoría y con aquellas otras que también lo estaban implementando. A continuación, se mencionan los aspectos que se consideran más relevantes de cada etapa, hasta dónde se ha avanzado, qué problemáticas se han presentado, sus soluciones, así como las interrogantes y los retos que han surgido.

\section{a) Actualización en RDA}

Desde que se anunció en los cursos y foros de actualización que ya no habría una $3^{a}$ edición de las RCA2 (Reglas de Catalogación Angloamericanas) surgió con más ímpetu la necesidad de una actualización constante por medio de la asistencia a foros nacionales e internacionales especializados en catalogación. Adicionalmente, se realizaron consultas directas con quienes que tenían información de primera mano y con quienes estaban directamente involucrados en el código RDA. La actualización se realizó a través del Instituto de Investigaciones Bibliotecológicas y de la Información (IIBI) por ser una dependencia de la Universidad Nacional Autónoma de México. Los cursos, conferencias y foros de discusión auspiciados y organizados por esta institución fueron factores que determinaron la decisión para poder implementar el "nuevo" código RDA. Otra forma de actualizarse de un modo directo fue con los asesores de los programas cooperativos internacionales, personal altamente calificado en la teoría y práctica en relación con este código, Adicionalmente, a través de la asistencia a los foros y los talleres impartidos sobre RDA.

La Asociación de Estados Iberoamericanos para el Desarrollo de las Bibliotecas Nacionales de Iberoamérica (ABINIA) jugó también un papel fundamental, porque apoyó la realización de un taller latinoamericano sobre control de autoridades cuyo instructor estaba certificado en los programas internacionales de catalogación cooperativa. A dicho taller asistieron representantes de diversas bibliotecas nacionales. Este evento también influyó para que la Biblioteca Nacional de México fuera miembro participante del programa cooperativo NACO, al que contribuyó con registros de autoridad de nombres personales.

La participación en este tipo de programas de cooperación trajo consigo grandes ventajas porque implicó estar actualizados con parámetros vigentes. Desafortunadamente, en 2011 se dejó de participar en el programa. Sin embargo, la actualización continuó porque la comunicación con colegas giraba en torno al nuevo código y, también, a través de la asistencia a cada curso o conferencia que se ofrecía al respecto. Cabe mencionar que para la actualización existió gran apoyo por parte de las autoridades de la institución, lo que dio pauta para que la implementación se diera casi un año después de que lo hicieran entidades responsables de la normalización bibliográfica en Australia, Canadá, Estados Unidos y Reino Unido, en 2013.

En la Biblioteca Nacional de México existe un cuerpo colegiado integrado por profesionales dedicados a la actividad de organización bibliográfica, en el cual están representados los responsables de catalogar los diversos tipos de recursos documentales y los integrantes de diferentes áreas. Los miembros del Comité de Catalogación de la Biblioteca Nacional de México, después de haber tomado los diferentes cursos de actualización, realizaron reuniones de trabajo exclusivamente para analizar y preparar la implementación de RDA. Las reuniones de trabajo duraron aproximadamente un año (2013) y en este lapso surgieron muchas dudas porque a pesar de que se habían tomado los cursos correspondientes se cuestionaba el por qué de la necesidad de cambiar del código RCA2 a RDA, sí en ese momento funcionaba bien el catálogo. Por otro lado, solo se tenía el estándar RDA en formato impreso y, cuando estuvo la 
herramienta disponible en línea, no se accedió de manera continua a ella. Asimismo, seguían surgiendo las preguntas referentes a todo lo que implicaba el cambio, pero especialmente a la interrogante de cómo comunicar a las autoridades de la institución que se iba a llevar dicho cambio, por lo que se concluyó que la mejor opción era estar seguros de qué era RDA, qué implicaría en el futuro, qué apoyos se necesitaban y qué beneficios inmediatos se irían presentando.

Después de las reuniones de análisis sobre RDA ya se contaban con más elementos para poder fundamentar la presentación ante las autoridades institucionales, por lo que se procedió a comunicar dicho cambio presentando las etapas en que se iba a trabajar. Afortunadamente, la respuesta fue favorable y se dieron los apoyos de la institución para iniciar la implementación. Cabe mencionar que al comunicarlo con las autoridades ya se tenían definidas las etapas en las cuales se iba a trabajar, las cuales fueron las siguientes:

- Cambios en el registro bibliográfico de cada tipo de recurso

- Plantillas o formatos para el ingreso de datos de cada tipo de recurso.

- Redacción de políticas y procedimientos.

- Trabajo de control de autoridades.

- Procedimiento para el control de autoridades.

- Requerimientos para el Departamento de Informática de cada etapa.

En lo que se refiere a la plataforma para el ingreso de datos, la Biblioteca Nacional lo realizó en el Sistema ALEPH 500, versión 21, y para ello se dieron de alta o se activaron las etiquetas que se solicitaron. No obstante, a pesar de que el sistema está preparado en el módulo de catalogación para soportar el formato MARC 21, el Departamento de Informática estaba en comunicación continua para ver los nuevos requerimientos.

Otra etapa que se tuvo que considerar fue la capacitación del personal. En este rubro, fue de gran ayuda la realización de plantillas para los diferentes tipos de recursos documentales y la redacción de políticas para realizar el trabajo de catalogación, ya que cuando se implementó RDA, la herramienta aún no se había traducido, lo que era una limitante para su consulta. La capacitación del personal ha sido continua por medio de talleres y pláticas. Sin embargo, en la práctica debe existir la supervisión o control de calidad para saber si se aplican los procedimientos y las políticas que se han establecido.

Los cambios principales que surgieron con la aplicación de RDA en la Biblioteca Nacional de México fueron:

- Aceptación de los acuerdos establecidos por el Comité de Catalogación, en cuanto a políticas para registrar los datos y delimitar hasta donde se registrarían dichos datos.

- Establecimiento de políticas en cuanto al registro de los puntos de acceso de nombres personales, de familia, entidades y títulos, así como los relacionados con la obra; así como los nuevos elementos relacionados con dichos puntos de acceso.

- Control de los puntos de acceso mediante registros de autoridades. 
- Nuevos elementos y datos para los registros de autoridades.

- Establecimiento de procedimientos y controles de trabajo para la elaboración de registro de autoridades.

- Consulta de la herramienta en línea.

\section{b) Impacto de la aplicación de RDA}

Los cambios antes mencionados han tenido un impacto en los siguientes aspectos: mayor inversión de tiempo, un trabajo más profesional, mayor apoyo tecnológico para el mantenimiento y actualización de la base bibliográfica. Respecto al control de autoridades, si bien se estuvo trabajando principalmente con nombres personales y materias de manera "manual", ahora representa una labor indispensable para que se pueda decir que se está aplicando RDA y verificar que los datos funcionen adecuadamente estableciendo las relaciones entre éstos. Sin embargo, como en todo cambio existen ventajas y desventajas. Respecto a las ventajas, el control de autoridades apoya en gran medida el trabajo diario de catalogación, pues es la herramienta principal para dicha actividad, ya que representa un nuevo concepto del control bibliográfico y repercute en la recuperación de información para cumplir con las tareas del usuario. Por otro lado, la base bibliográfica tiene mayor consistencia en cuanto a la uniformidad de los puntos de acceso, pero sobre todo, la recuperación de la información es más eficiente en relación con el registro de los datos de las variantes que se presentan y su establecimiento en los registros de autoridad.

Desde que el trabajo de control de autoridades se realizaba en registros de autoridad para nombres personales con datos que solo respondían a envíos y, para el caso encabezamientos de materia con envíos a términos generales o específicos, en el Departamento de Catalogación ya existía la Sección de Control de Autoridades, en la que solo había una única persona como responsable, realizando principalmente registros de autoridad de tema. Posteriormente, con la participación en el programa cooperativo NACO, también se realizaron registros de autoridad de nombres personales, todavía con RCA2, los que eran para la base bibliográfica pero también para la aportación a dicho programa.

Los registros de autoridad, a pesar de que eran con RCA2, se realizaron con datos que cumplían con parámetros funcionales para la organización y recuperación de información. A la par que se implementó RDA en los registros bibliográficos como primera etapa en el mes de abril de 2014, se continuó con la actualización sobre registros de autoridad y se preparó una segunda etapa para la aplicación del nuevo código con registros de autoridad, la que se inició en el mes de octubre de ese año. La preparación incluyó el diseño de plantillas con las etiquetas o campos que se necesitaban. Sin embargo, no se podía en ese momento pensar en lo que implicaba hacer un registro de autoridad con RDA.

La situación para realizar los registros de autoridad de la mayoría de puntos de acceso que incluyen cada uno de los registros bibliográficos es detallada; si bien se puede realizar con los elementos que trae el mismo recurso, dichos elementos no representan los datos o atributos suficientes para una autoridad, sobre todo cuando ya existen registros bibliográficos retrospectivos o que estaban en la base bibliográfica y que aportan variantes. Por lo que se ha optado por efectuar otras búsquedas para tener más datos, y esto ha dejado el registro de autoridad lo más completo posible. Este proceso implica mayor inversión de tiempo, tanto en la realización de registros como en la búsqueda de datos en la base bibliográfica. En cuanto a 
la supervisión, también implica mayor inversión de recursos humanos y tecnológicos porque se verifica el funcionamiento de los registros de autoridad para que estén ligados a los registros bibliográficos, y que las variantes de éstos funcionen en la búsqueda y recuperación de información.

Por otro lado, el registro de autoridad determina cuál va a ser el punto preferido de acceso de acuerdo al recurso que lo valida, aunque muchas veces es necesario actualizar los registros bibliográficos retrospectivos para que la base bibliográfica sea consistente en su funcionamiento. Por lo regular, esto se presenta con puntos de acceso actuales que no concuerdan con los retrospectivos -siendo esto común en una Biblioteca Nacional-, pero que son puntos de acceso que vale la pena trabajar porque pueden ser variantes que en la época en que se catalogaron no fueron consideradas. Cabe mencionar que cada tipo de registro de autoridad, como los de nombres personales, figura 1-2; nombres de entidades corporativas, figura3-4; nombres geográficos, figura 5; títulos de series, figura 6, incluyen elementos 0 datos que lo componen que pueden ser sencillos o complejos según sea el caso.

Figura 1. Registro de autoridad de nombre personal (Pantalla 1)

Seleccione formato: Estándar Nombres de etiquetas Etiquetas MARC
Registro 1 de 2
Número de sistema
Nombre autorizado
Variante

Figura 2. Registro de autoridad de nombre personal (Pantalla 2)

\begin{tabular}{|c|c|}
\hline Registro 2 de 2 & A Reg.anterior Regsigueinte - \\
\hline Número de sistema & 000082479 \\
\hline \multirow[t]{2}{*}{ Variante } & De Villavicencio, Pablo, 1796-1832 \\
\hline & op. V. \\
\hline Véase además & Pidentidad alternativa EI Payo del Rosario, 1796-1832 \\
\hline \multirow[t]{3}{*}{ Fuente } & El señor generalisimo penso acertarla y la erro, 1822:página 7 (Pablo de Villavicencio) \\
\hline & $\begin{array}{l}\text { Wikipedia, abr. 18, 2016:(Pablo de Villavicencio González; nació en EI Rosario, 1796; murió en Toluca, 1832; político y pensador mexicano, apodado El Payo del } \\
\text { Sinaloa)https://es. wikipedia.org/wiki/Pablo_de_Villavicencio }\end{array}$ \\
\hline & $\begin{array}{l}\text { Ruiz Castañeda, Maria del Carmen. Diccionario de seudónimos, anagramas, iniciales y otros alias usados por escritores mexicanos y extranjeros que han publicado en } \\
\text { México, 2000:página } 878 \text { (Villavicencio, Pablo de (1796-1832); n. en el mineral Real del Rosario, Provincia de Sonora, hoy estado de Sinaloa ; } m \text {. en Toluca, Edo, de } \\
\text { Mex.; E. P. D. R. [seud.] iniciales del seudónimo El Payo del Rosario) ; P. V. iniciales de su nombre) }\end{array}$ \\
\hline Liga & Villavicencio, Pablo den 83022432 \\
\hline Término & El Payo del Rosario,1796-1832 \\
\hline
\end{tabular}


Figura 3. Registro de autoridad de entidad corporativa (Pantalla 1)

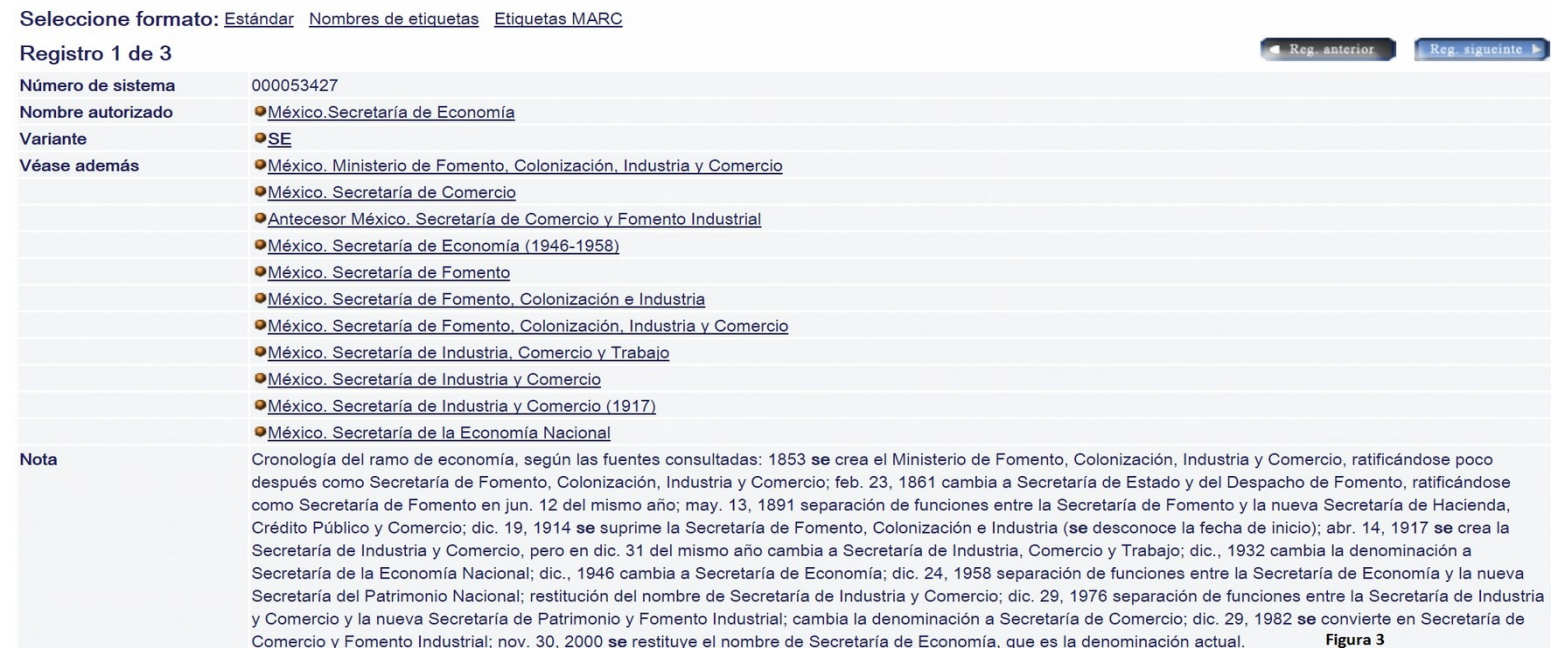

\section{Figura 4. Registro de autoridad de entidad corporativa (Pantalla 2)}

Fuente Economía 6 años de logros : un sexenio de esfuerzos..., 2006.portada (Secretaría de Economía, SE) verso de la portada (Secretaría de Economía) BNM. Base bibliográíica, ago. 26, 2014:(encab:: México. Secretaría de Economia)

Secretaria de Gobernación. Diario Oficial de la Federación. DOF: 19/07/2013. Manual de Organización General de la Secretaría de Economía. Documento electrónico, ago. 27, 2014:(Antecedentes: ....Mediante el Decreto por el que se reforman, adicionan y derogan diversas disposiciones de la Ley Orgánica de la Administración Pública Federal, publicada en el DOF el 30 de noviembre de 2000, la Secretaria de Comercio y Fomento Industrial cambió su denominación a la de Secretaría de Economía, adicionándole entre otros asuntos, el de coordinar y ejecutar la política nacional para crear y apoyar empresas que asocien a grupos de escasos recursos en áreas urbanas...)http://www.dof.gob. mx/nota_detalle. php?codigo=53074318fiecha=19/07/2013

SE, Secretaria de Economía. Sitio electrónico, ago. 27, 2014:(Misión.- Fomentar la productividad y competitividad de la economía mexicana mediante una innovadora politica de fomento industrial, comercial y de servicios, asi como el impulso a los emprendedores y las empresas de los sectores social y privado, fincado en la mejora regulatoria, la competencia de mercado y la diversificación del comercio exterior...; U Ubicación: Alionso Reyes №.30, Col. Hipódromo Condesa CP 06140, Del.

Cuauhtémoc México, Distrito Federal)http://luww.economia.gob. mx/conoce-la-se/mision-y-vision-se

LC Authorities, ene. 23, 2013:no 91030695 (Mexico. Secretaría de Economía)

Figura 4

Figura 5. Registro de autoridad de nombre geográfico

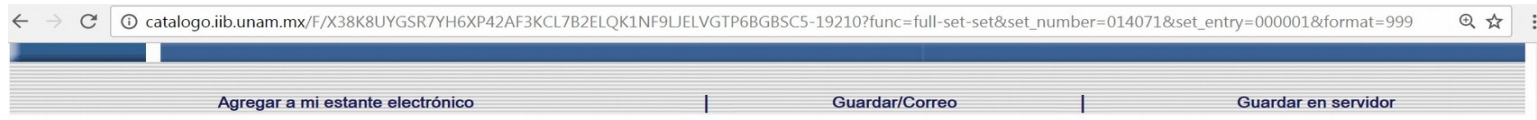

Vista del registro

Seleccione formato: Estándar Nombres de etiquetas Etiquetas MARC

Registro 1 de 1

Número de sistema

Parque Nacional Cofre de Perote (Veracruz)

aPNCP (Veracruz)

OPN Cofre de Perote (Veracruz)

Pance de Perte

- Nauhcampatépetl (Veracruz

Véase además $\quad$ Parques v reservas nacionales Méxice

Fuente Vázquez Ramirez, Jerónimo. Guia botánica del Parque Nacional Cofre de Perote, 2014:página 9 (PNCP)

Comisión Nacional de Areas Naturales Protegidas. Sitio electrónico, mayo 6, 2016:(PN Cofre de Perote O Nauhcampatépetl : comprende parte de los Municipios de

Perote, Xico, Ixhuacán de los Reyes y Ayahualulco, Estado de Veracruz ; Se encuentra localizado en las coordenadas geográficas, entre los paralelos de $19^{\circ} 25^{\prime} 33^{\prime \prime}$ a $19^{\circ} 33^{\prime} 52^{\prime \prime}$ de latitud norte y entre los meridianos de $97^{\circ} 06^{\prime} 55^{\prime \prime}$ a $97^{\circ} 12^{\prime} 52^{\prime \prime}$ de longitud oeste. El Parque Nacional Cofre de Perote, está delimitado por la curva de los 3,000 metros sobre el nivel del mar hasta su maxima elevacion que alcanza los 4,250 metros sobre el nivel del mar... En la epoca prehispánica esta montaña recibió el

Figura 5

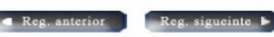




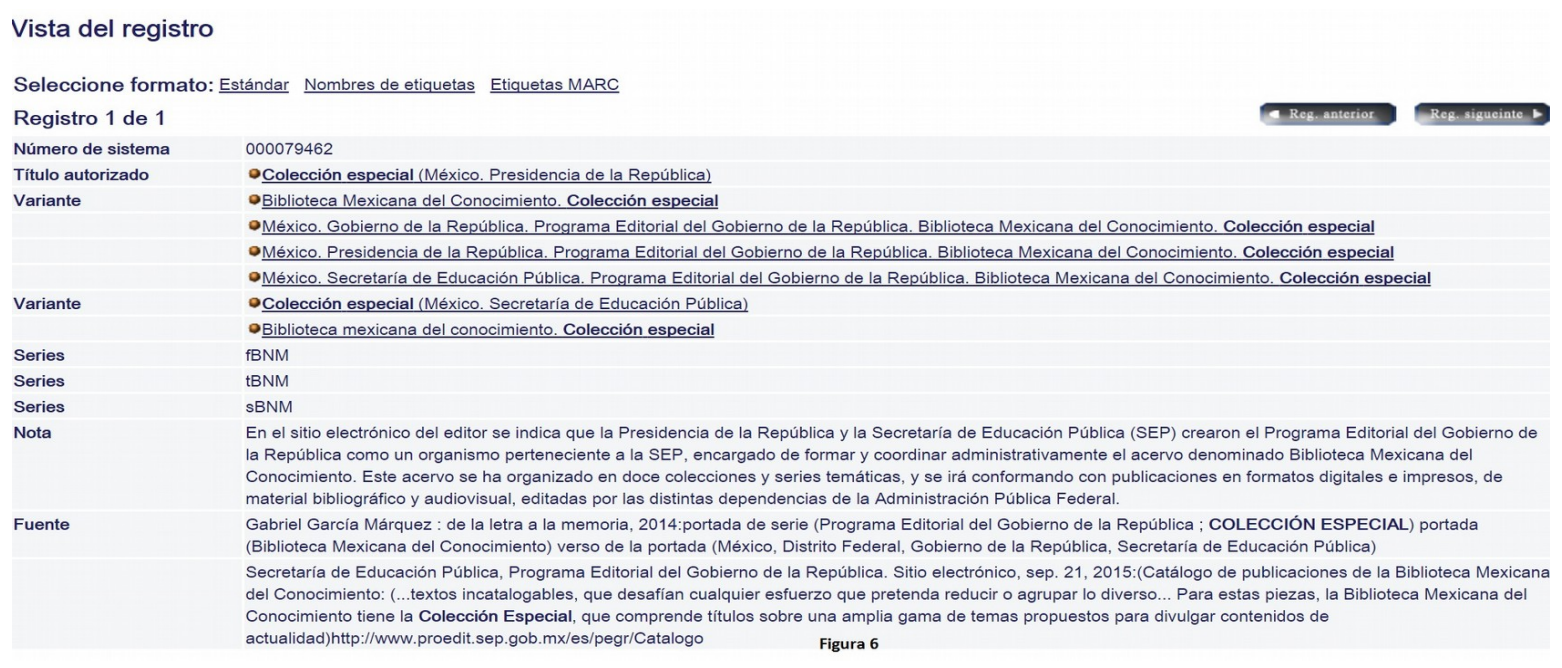

Los registros de autoridad pueden tener datos que se registran en etiquetas específicas a las que se nombran variantes, las que hacen posible la recuperación de información por las diversas formas en las que se busca. La Biblioteca Nacional de México, por su naturaleza de conservar documentos de diferentes épocas, permite tener datos que pueden registrarse en las autoridades con toda certeza para ser validados.

El trabajo de control de autoridades es costoso, aunque se ha mencionado que los programas cooperativos pueden apoyar esta actividad, pues solo hay que bajar los registros y hacer las adecuaciones correspondientes. No obstante, la experiencia no ha sido positiva, porque más bien estos catálogos solo representan referencias o consultas que se pueden realizar a través de ellos, pues en el caso de las bibliotecas nacionales son ellas mismas las que podrían aportar esa información, sobre todo porque por lo regular son las depositarias legales. Por otro lado, las bibliotecas de América Latina, hasta el momento, no ofrecen acceso a sus catálogos de autoridad por vía remota, incluso, algunas de ellas no efectúan el trabajo de control de autoridades. Por otro lado, se considera que con tener acceso a un catálogo como el de la Biblioteca del Congreso, por mencionar un ejemplo, se tiene resuelto este rubro. Sin embargo, en la práctica no resulta del todo eficiente, ya que no se trata de copiar y pegar datos, hay que analizar muy bien los que se quitan y se agregan para que, realmente, el registro de autoridad represente la validez del recurso que tiene la biblioteca que lo está aportando.

Hasta ahora, en el caso de América Latina, solo la Biblioteca Nacional de México y la Dirección de Bibliotecas de la Universidad Nacional Autónoma de México son las que ofrecen consulta pública a sus catálogos de autoridad. Puesto que la actividad del control de autoridades representa una de las que requieren mayor trabajo, se espera que estos catálogos sean de utilidad para otras bibliotecas y apoyen la creación de relaciones entre los puntos de acceso de los registros bibliográficos.

Otro problema que se ha presentado es con respecto al apoyo tecnológico por parte del equipo de trabajo de informática, ya que si bien se ha realizado lo pertinente para la implementación de RDA, se necesita mayor apoyo para concretar adecuaciones o resolver situaciones y requerimientos a los que el sistema no está respondiendo. Por otro lado, la plataforma tecnológica hasta el momento no ha podido efectuar las relaciones que promete RDA, solo se han estado organizando los datos con MARC 21 de la manera más consistente, pero es de 
esperarse que los profesionales del área de sistemas o la tecnológica hagan lo correspondiente para efectuar las relaciones de RDA.

\section{c) Retos}

Los retos que ha traído consigo la adopción e implementación de RDA son los siguientes:

- Que los todos los registros bibliográficos cuenten con la mayor parte de puntos de acceso controlados mediante un registro de autoridad.

- Que los registros de autoridad y bibliográficos tengan los elementos y/o datos consistentes para que puedan interactuar con las plataformas tecnológicas.

- Que los registros de autoridad y bibliográficos retrospectivos no presenten problemas a futuro para la migración a otras plataformas de almacenamiento y recuperación de información.

- Que el OPAC tenga un funcionamiento y diseño adecuado para la búsqueda y recuperación de información y pueda cumplir con las tareas del usuario.

- Que la institución tenga los recursos económicos para la adquisición actualización de la plataforma o tecnología adecuada para cumplir con lo planteado por RDA.

- Que se efectúen estudios de usuarios con la finalidad de conocer si los elementos que estamos registrando son de ayuda en sus necesidades de información.

\section{d) Consideraciones en torno a la aplicación de RDA}

- La Biblioteca Nacional de México ha continuado su trabajo de catalogación con la normatividad vigente esperando ser un referente para dar pautas o directrices para el control bibliográfico del país.

- Decidió aplicar el nuevo código en el año 2014, un año después de que las instituciones que llevaban la vanguardia lo hicieran.

- La toma de decisiones para la aplicación fue acordada de manera colegiada a través de Comité de Catalogación de la Biblioteca Nacional de México.

- Las autoridades institucionales apoyaron el cambio a través de facilidades para la capacitación del personal y los recursos tecnológicos.

- La comunicación estrecha con el Departamento de Informática tuvo que mantenerse en todo momento en buenos términos para lograr dicha implementación.

- La actualización y capacitación del personal debe estar en todo momento presente con la finalidad de facilitar el cambio.

- Aplicar RDA implica tener una suscripción de la herramienta en línea, lo cual también es un cambio sustancial en comparación con la normatividad anterior.

- Con la implementación de RDA se ha incrementado el trabajo debido al control de autoridades, lo cual requiere además de mayor inversión de tiempo, así como una constante actualización y edición de registros bibliográficos. 
- Queda claro que el control de autoridades es primordial para el trabajo de catalogación y un nuevo énfasis para el control bibliográfico.

- La Biblioteca Nacional de México ha puesto a disposición de otras bibliotecas la consulta de sus registros de autoridad, para así cumplir en parte con su misión de órgano bibliográfico nacional.

- Se espera que la plataforma tecnológica responda a la organización y recuperación de información tal como lo plantea RDA.

\section{Implementación de RDA en la Dirección General de Bibliotecas}

Como se ha mencionado, los materiales documentales adquiridos por las bibliotecas que integran el Sistema Bibliotecario y de Información de la UNAM (SIBIUNAM) son procesados técnicamente por la DGB con el sistema de clasificación de la Biblioteca del Congreso de Estados Unidos (LC) y las normas RDA (Recursos, Descripción y Acceso). La riqueza documental de SIBIUNAM se puede localizar mediante los catálogos colectivos que reúnen los recursos existentes en todas las unidades que lo conforman, siendo uno de los principales LIBRUNAM, el cual da cuenta de los más de 1.700 .000 libros existentes en las diferentes bibliotecas de SIBIUNAM. Durante 2015, la colección de libros del Sistema Bibliotecario creció en más de 70.000 títulos impresos y 7.405 libros electrónicos (UNAM, 2016), los cuales tuvieron que ser catalogados e integrados al catálogo LIBRUNAM, que puede ser consultado en: $\quad$ http://librunam.dgbiblio.unam.mx:8991/F/?func=find-b-0\&local base=mx001. Adicionalmente, los catálogos de otros tipos de recursos que la DGB desarrolla se pueden acceder en: http://bibliotecas.unam.mx/index.php/catalogos.

La Dirección General de Bibliotecas (DGB) de la Universidad Nacional Autónoma de México (UNAM) ha estado siempre a la vanguardia en los procesos de catalogación y clasificación, teniendo como principio aplicar las normas internacionales. A través de la historia, en esta institución se han aplicado diversos estándares de catalogación, así fue que las Reglas de Catalogación Angloamericanas $1^{\text {a }}$ edición (RCA) fueron utilizadas hasta el año 1985, fecha en la que se inicia la aplicación de las Reglas de Catalogación Angloamericanas $2^{a}$ edición (RCA2) para la descripción bibliográfica de sus materiales.

Con la llegada del código de organización de la información denominado RDA (Resource Description and Access) -el cual rompe con el modelo de la organización bibliográfica, basándose en el modelo conceptual Functional Requirements for Bibliographic Records (FRBR) desarrollado por la International Federation of Library Associations and Institutions (IFLA)- se considera la viabilidad de aplicarlo en los registros del Catálogo del Sistema Bibliotecario y de la Información de la UNAM (SIBIUNAM), LIBRUNAM. Es así como se inicia el análisis del mencionado código y el estudio de la situación que hasta en ese momento guardaban los registros de LIBRUNAM, al mismo tiempo que se considera la necesidad de corregir ciertas inconsistencias que hasta ese momento prevalecían en dicho catálogo, y contemplar además la aplicación del código RDA.

En marzo de 2010 empiezan las modificaciones necesarias para elevar la uniformidad del catálogo LIBRUNAM, efectuando de manera global cambios como la corrección de usar solo mayúsculas a usar mayúsculas y minúsculas de títulos y nombres de autores y de entidades corporativas mal escritos, así como la unificación de las diferentes formas de estos nombres a una forma única, además de la conversión de la etiqueta 008 en formato MARC-DGB a 
MARC21, entre otros. Es importante mencionar que desde 1996, el software utilizado en la DGB es el sistema integral para bibliotecas ALEPH en sus diferentes versiones. En 2013 se inicia la migración a la versión 21 utilizada actualmente, la cual cubre adecuadamente las necesidades de conversión de las reglas aplicadas a la catalogación de los materiales bibliográficos con que cuenta el Sistema Bibliotecario y de la Información de la UNAM (SIBIUNAM) (Granados Xolalpa, E., Mendoza Benítez, L., 2014). Una de las características de este sistema es la posibilidad de realizar cambios globales, es decir, la modificación de varios registros del catálogo en forma masiva.

Aunado a lo anterior se realizó el análisis de las cualidades y características de RDA para contrastarlas con las características de los registros bibliográficos de LIBRUNAM. En este sentido se menciona de manera resumida que RDA sirve para indicar atributos y relaciones de entidades, por lo que está constituido por tres elementos principales:

1. Las entidades: objetos físicos o abstractos,

2. Los atributos: propiedades o características de cada entidad y

3. Las relaciones: las interacciones que existen entre los datos de cada entidad del modelo. (Rodríguez García, 2012)

Además, RDA se basa en el modelo FRBR y en los principios del Statement of International Cataloguing Principles, ICP por sus siglas en inglés. El código RDA es un conjunto de estándares basados en principios que facilitan el proceso de descripción, diseñado para usarse fácilmente y de esta forma permitir la generación de registros con datos relevantes para los usuarios. También está orientado al contenido, por lo indican cómo describir un recurso y cómo registrar los atributos más conocidos por los usuarios. Estos lineamientos están orientados a la estandarización en el ambiente digital (Rodríguez García, 2012). De esta manera, sus principios se basan fundamentalmente en la representación. Por otra parte, sus objetivos para identificar y relacionar se centran en satisfacer al usuario.

RDA aborda la construcción de puntos de acceso autorizados y las variantes de éstos para las obras, expresiones, personas, familias y organismos corporativos, además de establecer relaciones de los siguientes tipos: relaciones entre la obra, la expresión, la manifestación y el ítem. Asimismo, las relaciones entre un recurso y las personas, familias y entidades corporativas asociadas a éste, así como las relaciones entre las personas, familias y organismos corporativos.

Este código prescribe una serie de elementos núcleo, los cuales son un conjunto de elementos mínimos que deben contener los registros. Es importante mencionar que en RDA se toma la información tal y como aparece en la fuente, por lo que regularmente no se usan abreviaturas; además se produce un cambio en la terminología, por ejemplo en el caso de autor a creador, o el caso de los asientos principales y secundarios a puntos de acceso.

Con el estudio y análisis de las características de RDA, señaladas anteriormente de manera general, la DGB, a través de su Departamento de Procesos Técnicos, inició en marzo de 2010 diversos cambios en el catálogo LIBRUNAM de manera global. Las conversiones de RCA2 al código RDA en sus registros bibliográficos se mencionan a continuación: 
a) Título preferido

\begin{tabular}{|c|c|c|}
\hline RDA & MARC21 & OBSERVACIONES \\
\hline $\begin{array}{l}\text { Título preferido. Instrucción general para la } \\
\text { elección del título preferido. (Obras musicales, } \\
\text { legales, religiosas y comunicaciones oficiales). } \\
\text { 6.2.1. Instrucciones generales; } 6.2 .2 \text { Título } \\
\text { preferido de la obra; } 6.2 .2 .1 \text { Alcance; } 6.2 .2 .3 \\
\text { Instrucción general para la elección del título } \\
\text { preferido; } 6.14 .2 \text { Obras musicales; } 6.19 .2 \\
\text { Obras legales; } 6.23 .2 \text { Obras religiosas; } 6.26 .2 \\
\text { Comunicaciones oficiales }\end{array}$ & $\begin{array}{l}\text { Etiquetas: 130; } \\
240 ; \\
730 \\
\text { Subcampo |a }\end{array}$ & $\begin{array}{l}\text { Cambio en la } \\
\text { terminología de } \\
\text { título uniforme a } \\
\text { título preferido }\end{array}$ \\
\hline
\end{tabular}

En este caso se han utilizado los títulos preferidos para la Biblia, obras legales y para las obras clásicas y completas. Con RDA, los títulos específicos de los libros de la Biblia se proporcionan de manera directa, sin subordinación al testamento correspondiente. En el caso de las obras completas de autores de la literatura se agregó la etiqueta 240, con el título preferido Obras (García López, Mejía Ruiz, Ramos Díaz \& Guzmán Rodríguez, E. M., 2012, p. 44).

b) Variante del título

\begin{tabular}{|c|c|c|}
\hline RDA & MARC21 & OBSERVACIONES \\
\hline $\begin{array}{l}\text { Inexactitudes } \\
\text { Transcriba un elemento como aparece en la } \\
\text { fuente de información, transcriba la inexactitud } \\
\text { o palabra mal escrita como aparece en la } \\
\text { fuente, excepto cuando se indique lo contrario. } \\
\text { Si la inexactitud aparece en el título, registre la } \\
\text { forma correcta del título como un título } \\
\text { variante, ver } 2.3 .6 \text {, si es considerado } \\
\text { importante para identificarlo. } 1.7 .9 \text {. } \\
\text { Transcriba el título como aparece en la fuente } \\
\text { de información. } 2.3 .1 .4\end{array}$ & $\begin{array}{l}246 ; \\
740 \\
\text { Subcampo: |a }\end{array}$ & $\begin{array}{l}\text { Nota: No corregir } \\
\text { errores para } \\
\text { monografías. No } \\
\text { utilizar [sic] o [i.e.], } \\
\text { no se indican notas } \\
\text { explicativas al } \\
\text { respecto. }\end{array}$ \\
\hline
\end{tabular}

Variante del título: se agrega en tres situaciones: cuando una misma obra aparece con diferentes títulos, cuando el título contiene una inexactitud y cuando obras del mismo autor están encuadernadas en el mismo volumen. Etiquetas 246 y 740 de MARC21. (García López, Mejía Ruiz, Ramos Díaz y Guzmán Rodríguez, E. M., 2012, p. 50-51). 
c) Mención de responsabilidad

\begin{tabular}{|c|c|c|}
\hline RDA & MARC21 & OBSERVACIONES \\
\hline $\begin{array}{l}\text { Registre una mención de responsabilidad } \\
\text { indicando más de una persona ya sean } \\
\text { personas, familias o entidades corporativas que } \\
\text { realicen la misma función o diferentes. } \\
\text { Opción. A juicio del catalogador transcribir el } \\
\text { primer nombre y dar entre corchetes la } \\
\text { información no mencionada, indicar [y otros]. } \\
2.4 .1 .5\end{array}$ & $\begin{array}{l}\text { Etiqueta: } 245 \\
\text { Subcampo: |c }\end{array}$ & $\begin{array}{l}\text { Queda fuera la regla } \\
\text { de tres. } \\
\text { Si se aplica la } \\
\text { optativa: no se } \\
\text { indica: }[\text { et al.] } \\
\text { Se indica: }[y \text { otros] }\end{array}$ \\
\hline
\end{tabular}

En este caso, todos aquellos registros que indicaban "[et al.]" se modificaron como lo indica RDA y se cambiaron a "[y otros]". Asimismo, a partir de la aplicación del nuevo código se indican todos los creadores/colaboradores mencionados en la fuente.

\section{d) Designación de edición}

\begin{tabular}{|l|l|l|}
\hline RDA & MARC21 & OBSERVACIONES \\
\hline $\begin{array}{l}\text { Designación de la edición } \\
\begin{array}{l}\text { Una designación de edición es una palabra, } \\
\text { carácter, o grupo de palabras y/o caracteres } \\
\text { que identifican la edición del recurso que se } \\
\text { describe. } 2.5 .2 ; 2.5 .2 .1\end{array}\end{array}$ & $\begin{array}{l}\text { Etiqueta: } 250 \\
\text { Subcampo: Ia }\end{array}$ & $\begin{array}{l}\text { Nota: Transcriba } \\
\text { como se encuentre, } \\
\text { no utilizar } \\
\text { abreviaturas, no } \\
\text { convertir a } \\
\text { numerales }\end{array}$ \\
\hline
\end{tabular}

De acuerdo a lo que dicta el código ya no se utilizan abreviaturas, se modifican todos los registros indicando la versión completa de las mismas. Actualmente se toman las palabras relacionadas con la edición, tal y como se encuentran en la fuente.

\section{e) Lugar de publicación no identificado}

\begin{tabular}{|l|l|l|}
\hline RDA & MARC21 & OBSERVACIONES \\
\hline $\begin{array}{l}\text { Lugar de publicación no identificado en el } \\
\text { recurso }\end{array}$ & $\begin{array}{l}\text { Etiqueta: } 264 \\
\text { Si no se conoce un lugar probable como país, } \\
\text { estado, provincia, etc., de publicación que }\end{array}$ & $\begin{array}{l}\text { Si no hay un } \\
\text { probable lugar de } \\
\text { publicación no } \\
\text { indicar [s.l.] }\end{array}$ \\
\hline
\end{tabular}


pueda ser determinado, registrar [Lugar de publicación no identificado]

\subsubsection{6}

Se indica: [Lugar de publicación no identificado]

Anteriormente se utilizaba la etiqueta 260, sin embargo, para detallar y relacionar los datos de publicación se utiliza la etiqueta 264. Todos los registros que no tenían un lugar de publicación identificado se modificaron a "[Lugar de publicación no identificado]".

\section{f) Editorial}

\begin{tabular}{|l|l|l|}
\hline RDA & MARC21 & OBSERVACIONES \\
\hline $\begin{array}{l}\text { Transcriba los lugares de publicación y los } \\
\text { nombres de las editoriales en la forma en la } \\
\text { cual aparece en la fuente de información. } \\
\begin{array}{l}\text { Aplicar las guías generales sobre } \\
\text { transcripción indicadas en } 1.7 .\end{array}\end{array}$ & $\begin{array}{l}\text { Etiqueta: } 264 \\
\text { Subcampo: lb }\end{array}$ & $\begin{array}{l}\text { La editorial se } \\
\text { transcribe tal y como } \\
\text { aparece la editorial } \\
\text { en la fuente, no hay } \\
\text { omisión de palabras. }\end{array}$ \\
\hline
\end{tabular}

Se unificaron todos los registros a una misma forma del nombre de la editorial, sin embargo, a partir de la introducción de RDA se transcriben tal y como aparecen, como se indica.

\section{g) Editorial no identificada}

\begin{tabular}{|l|l|l|}
\hline RDA & MARC21 & OBSERVACIONES \\
\hline $\begin{array}{l}\text { Para un recurso publicado en una sola } \\
\text { forma, si no existe un nombre de editorial y } \\
\text { no se puede identificar por otras fuentes } \\
\text { como se especifica en 2.2.4, registrar } \\
\text { [Editorial no identificada]. 2.4.8.7 }\end{array}$ & $\begin{array}{l}\text { Etiqueta: } 264 \\
\text { Subcampo: } \mid \mathrm{b}\end{array}$ & $\begin{array}{l}\text { Si no hay una } \\
\text { editorial identificada } \\
\text { no indicar [s.n.] }\end{array}$ \\
& & $\begin{array}{l}\text { Indicar: [Editorial no } \\
\text { identificada] }\end{array}$ \\
\hline
\end{tabular}

Todos aquellos registros que no tenían una editorial identificada se corrigieron para indicar: [Editor no identificado]. 
h) Fecha de publicación

\begin{tabular}{|l|l|l|}
\hline RDA & MARC21 & OBSERVACIONES \\
\hline $\begin{array}{l}\text { Si no hay una fecha estimada del recurso } \\
\text { registrar [Fecha de publicación no } \\
\text { identificada] } \\
2.10 .6 .3\end{array}$ & $\begin{array}{l}\text { Etiqueta 264 } \\
\text { Subcampo: Ic }\end{array}$ & $\begin{array}{l}\text { No se dan décadas } \\
\text { probables ni } \\
\text { aproximadas } \\
\text { Se indica: [Fecha de } \\
\text { publicación no } \\
\text { identificada] }\end{array}$ \\
\hline
\end{tabular}

Todos los registros que no tenían una fecha identificada se modificaron a: [Fecha de publicación no identificada]

\section{i) Descripción física}

\begin{tabular}{|l|l|l|}
\hline RDA & MARC21 & OBSERVACIONES \\
\hline $\begin{array}{l}\text { Registre el número exacto de páginas, si } \\
\text { puede ser fácilmente determinado (93 } \\
\text { páginas sin numerar). }\end{array}$ & $\begin{array}{l}\text { Etiqueta: } 300 \\
\text { Subcampo: } \mid \text { la }\end{array}$ & $\begin{array}{l}\text { No se usan } \\
\text { abreviaturas, se } \\
\text { indica: páginas, } \\
\text { volúmenes, hojas }\end{array}$ \\
$\begin{array}{l}\text { Si no es fácil determinar el número, registrar } \\
\text { un número estimado de páginas, hojas o } \\
\text { columnas precedido por aproximadamente } \\
\text { (aproximadamente 600 páginas) }\end{array}$ & $\begin{array}{l}\text { No se usa: c.a.; } \\
\text { Registre 1 volumen sin numerar. }\end{array}$ & $\begin{array}{l}\text { Se usa } \\
\text { aproximadamente } \\
\text { No se usa: i.e. }\end{array}$ \\
\hline
\end{tabular}

En este campo, dentro de todos los registros se modificaron las abreviaturas a su forma completa como lo indica RDA, por ejemplo, "p." por "páginas", "il." por "ilustraciones", y en general todas las abreviaturas.

\section{j) Creador}

\begin{tabular}{|l|l|l|}
\hline RDA & MARC21 & OBSERVACIONES \\
\hline Creador= persona, familia o entidad & Etiquetas: & Cambio en la \\
\hline
\end{tabular}


corporativa responsable de la creación de una obra

19.2
$1 X X$

$7 X X$

Subcampo |a; |

e terminología de autor a creador.

En este campo, dentro de todos los registros se agregó la función autoren las etiquetas $1 \mathrm{XX}$ y en la etiqueta 7XX se cambiaron de coautor a autor)

k) Abreviaturas en los designadores de relación

\begin{tabular}{|c|c|c|}
\hline RDA & MARC21 & OBSERVACIONES \\
\hline $\begin{array}{l}\text { Abreviaturas: aplicar las instrucciones sobre el } \\
\text { uso de abreviaturas indicadas en el apéndice } \\
\text { (B.4.). } 1.7 .8 \\
\text { Al transcribir los elementos use únicamente las } \\
\text { abreviaturas encontradas en la fuente de } \\
\text { información. En todos los demás casos } \\
\text { generalmente no se abrevian las palabras. } \\
\text { Apéndice B.4 } \\
\text { Designador de relación } \\
\text { Registre uno o más términos apropiados listados } \\
\text { en el apéndice I, para identificar los puntos de } \\
\text { acceso autorizados que represente una persona, } \\
\text { familia o entidad corporativa para indicar la } \\
\text { naturaleza de la relación más específicamente } \\
\text { como esté indicado para definir el alcance de la } \\
\text { relación entre los elementos. } \\
\text { Autor, productor, director, actor, compositor, } \\
\text { cartógrafo, impresor, organismo emisor, } \\
\text { conductor, artista, etc. 18.5.1.3 }\end{array}$ & $\begin{array}{l}\text { Etiquetas: } \\
1 X X ; \\
7 X X \\
\text { Subcampo: } \\
\text { le }\end{array}$ & $\begin{array}{l}\text { Las funciones se dan } \\
\text { de manera completa, } \\
\text { no se usan } \\
\text { abreviaturas } \\
\text { RDA incluye un } \\
\text { listado para } \\
\text { identificar los puntos } \\
\text { de acceso } \\
\text { autorizados en el } \\
\text { cual se menciona: } \\
\text { autor, productor, } \\
\text { director, actor, } \\
\text { compositor, } \\
\text { cartógrafo, impresor, } \\
\text { conductor, artista, } \\
\text { etcétera. }\end{array}$ \\
\hline
\end{tabular}

En este caso, dentro de todos los registros se cambiaron las abreviaturas correspondientes a las funciones por la versión completa en las etiquetas 1XX y 7XX. También se determinaron las designaciones de relación de los organismos con la obra, mencionados en la etiqueta 500, e indicados en la etiqueta MARC 710. (García López, Mejía Ruiz, Ramos Díaz y Guzmán Rodríguez, E. M., 2012, p. 31). 
I) Designación General del Material

\begin{tabular}{|l|l|l|}
\hline RDA & MARC21 & OBSERVACIONES \\
\hline $\begin{array}{l}\text { Tipo de contenido } \\
\text { Tipo de medio }\end{array}$ & $\begin{array}{l}\text { Etiqueta: 336 } \\
\text { Subcampo: |a término; |2 } \\
\text { Tipo de soporte }\end{array}$ & $\begin{array}{l}\text { La designación } \\
\text { general del material } \\
\text { indicada en RCA2, } \\
\text { dentro de MARC se } \\
\text { registra en los campos } \\
\text { fijos. }\end{array}$ \\
& $\begin{array}{l}\text { Etiqueta 337 } \\
\text { Subcampo: |a término |2 } \\
\text { RDAmedia } \\
\text { Etiqueta 338 } \\
\text { Subcampo: |a término |2 } \\
\text { RDAcarrier }\end{array}$ & \\
\hline & & \\
\hline
\end{tabular}

Se adecuó la plantilla MARC21 para monografías y se agregaron las etiquetas 336, 337 y 338 con sus correspondientes subcampos, de la siguiente manera:

Etiqueta: 336. Subcampo: |a texto; 12 rdacontent

Etiqueta 337. Subcampo: |a sin medio 12 rdamedia

Etiqueta 338. Subcampo: |a volumen 12 rdacarrier

Para el caso de las monografías electrónicas en línea las etiquetas 336, 337 y 338 quedaron como sigue:

Etiqueta: 336. Subcampo: |a texto; 12 rdacontent

Etiqueta 337. Subcampo: |a computadora 12 rdamedia

Etiqueta 338. Subcampo: |a recurso en línea $\mid 2$ rdacarrier

Más una etiqueta de término género-forma: 6557 |a Libros electrónicos |2 local

Adicionalmente, algunos otros cambios que se han efectuado en los registros de LIBRUNAM son los mencionados a continuación:

Una modificación importante es la que se refiere a la etiqueta 040 a la cual se le agregó el subcampo le para señalar que se trata de un registro rda.

Actualmente se continúa realizando el análisis de los registros bibliográficos y se adaptan en MARC21 los cambios de RCA2 a RDA.

En el caso del catálogo de autoridad, para las autoridades de nombres de autores se empezó a trabajar en la conversión a RDA en octubre de 2011 y se han agregado los siguientes elementos:

Etiqueta 371, subcampos |a; |c; |d. Indica: Dirección 
Etiqueta 373, subcampo |a. Indica: Afiliación

Etiqueta 375, subcampo |a. Indica: Género

Por otro lado, el Departamento de Publicaciones Periódicas y Mapas coordina y controla la actualización del catálogo colectivo SERIUNAM, el cual contiene los registros de las publicaciones seriadas de 120 bibliotecas de la UNAM que poseen este tipo de colecciones, y de otras 238 bibliotecas de diversas instituciones de educación superior mexicanas. En este catálogo también se ha realizado el análisis para la adecuación de la plantilla MARC21 a la incorporación de RDA.

\begin{tabular}{|c|c|c|}
\hline RDA & MARC21 & Adecuación a SERIUNAM \\
\hline Fuente de catalogación & 040 & $\begin{array}{l}\text { Se hace referencia al código de } \\
\text { catalogación utilizado |erda }\end{array}$ \\
\hline Título preferido & $130 ; 240$ & $\begin{array}{l}\text { Utilizada para la catalogación de las } \\
\text { publicaciones seriadas electrónicas }\end{array}$ \\
\hline Título propio & $\begin{array}{c}\text { 245; subcampo } \\
\text { Ih }\end{array}$ & $\begin{array}{c}\text { La Designación General del Material (DGM) } \\
\text { da pauta a la implementación de las } \\
\text { etiquetas } 336 ; 337 \text { y } 338\end{array}$ \\
\hline Datos de publicación & 264 & $\begin{array}{c}\text { Se eliminaron locuciones latinas y } \\
\text { abreviaturas. Anteriormente se utilizaba la } \\
\text { etiqueta } 260 \text {, sin embargo, para detallar y } \\
\text { relacionar los datos de publicación se utiliza } \\
\text { la etiqueta } 264 \text {. }\end{array}$ \\
\hline Rdacontent & 336 & $\begin{array}{c}\text { Implementación de estas etiquetas para } \\
\text { sustituir DGM; } 245 \text { lh }\end{array}$ \\
\hline Rdamedia & 337 & \\
\hline rdacarrier & 338 & \\
\hline Fuente de descripción & 588 & $\begin{array}{c}\text { Descripción basada; y, último ejemplar } \\
\text { consultado, anteriormente utilizadas en la } \\
\text { etiqueta } 500\end{array}$ \\
\hline
\end{tabular}


También es conveniente mencionar que MAPAMEX es el catálogo colectivo que contiene las colecciones de mapas de 13 bibliotecas del SIBIUNAM y de 8 mapotecas de instituciones de educación superior y de Instituciones gubernamentales del país. En relación a este catálogo, las modificaciones necesarias para pasar a RDA se iniciaron en septiembre de 2016; se realizaron cambios globales a sus registros y adecuaciones a su plantilla, los que a continuación se mencionan:

\begin{tabular}{|c|c|c|}
\hline RDA & MARC21 & Adecuación a SERIUNAM \\
\hline $\begin{array}{c}\text { Fuente de } \\
\text { catalogación }\end{array}$ & 040 & $\begin{array}{c}\text { Se hace referencia al código de } \\
\text { catalogación utilizado |erda }\end{array}$ \\
\hline Título preferido & $130 ; 240$ & $\begin{array}{l}\text { Utilizada para la catalogación de las } \\
\text { publicaciones seriadas electrónicas }\end{array}$ \\
\hline Título propio & $\begin{array}{c}\text { 245; } \\
\text { subcampo |h }\end{array}$ & $\begin{array}{l}\text { La Designación General del Material } \\
\text { (DGM) da pauta a la implementación de } \\
\text { las etiquetas } 336 ; 337 \text { y } 338\end{array}$ \\
\hline $\begin{array}{l}\text { Datos de } \\
\text { publicación }\end{array}$ & 264 & $\begin{array}{c}\text { Se eliminaron locuciones latinas y } \\
\text { abreviaturas. Anteriormente se utilizaba } \\
\text { la etiqueta } 260, \text { sin embargo, para } \\
\text { detallar y relacionar los datos de } \\
\text { publicación se utiliza la etiqueta } 264 \text {. }\end{array}$ \\
\hline Rdacontent & 336 & Implementación de estas etiquetas para \\
\hline $\begin{array}{l}\text { Rdamedia } \\
\text { rdacarrier }\end{array}$ & $\begin{array}{l}337 \\
338\end{array}$ & \\
\hline $\begin{array}{l}\text { Fuente de } \\
\text { descripción }\end{array}$ & 588 & $\begin{array}{c}\text { Descripción basada; y, último ejemplar } \\
\text { consultado, anteriormente utilizadas en } \\
\text { la etiqueta } 500\end{array}$ \\
\hline
\end{tabular}

Finalmente, es importante mencionar que los cambios realizados en los registros de los tres catálogos de la DGB, anteriormente mencionados, presentan grandes avances, aunque aún se seguirá trabajando en realizar el análisis correspondiente a cada uno de ellos para la conversión y aplicación completa de RDA. También es importante mencionar que desde el primer trimestre de 2016 se están agregando de manera paulatina los campos de las relaciones para obtener todas las ventajas que ofrece este nuevo código de catalogación. Actualmente se están estableciendo las relaciones bibliográficas del grupo 1 de FRBR con la finalidad de que el usuario pueda identificar de forma explícita las relaciones entre obra-obra, expresión-expresión y manifestación-manifestación para así ampliar sus opciones de búsqueda y recuperación.

El Instituto de Investigaciones Bibliotecológicas y de la Información y la implementación de RDA

El Instituto de Investigaciones Bibliotecológicas y de la Información (IIBI), desde su creación como Centro Universitario de Investigaciones Bibliotecológicas (CUIB), ha fomentado el acercamiento entre la investigación y los bibliotecólogos que dedican gran parte de su quehacer profesional a la práctica profesional. En este sentido, sus proyectos y actividades del 
área de la organización de la información han abordado la problemática de la adopción e implementación de las RDA.

Una de las áreas de investigación del IIBI es la de Organización de la información y el conocimiento, la cual se encarga de estudiar "las teorías y principios de la representación, organización y recuperación de la información y el conocimiento, además de los fenómenos relacionados con la lingüística documental y la terminología en las estructuras del conocimiento" (IIBI UNAM, 2017). En esta área se incluyen cuatro líneas de investigación: Normalización de estándares; Lenguajes de indización; Sistematización y organización; Análisis y representación de contenido; dentro de ellas se desarrollan dos proyectos relacionados con RDA: "Impacto de las RDA (Resource Description and Access) en los sistemas de recuperación de la información", por el Dr. Filiberto Felipe Martínez Arellano y "Estandarización de los elementos bibliográficos en el entorno de la descripción y acceso a los recursos", por el Dr. Ariel Alejandro Rodríguez García.

\section{a) Encuentros de Catalogación y Metadatos}

Por otra parte, desde los inicios del surgimiento del nuevo estándar de catalogación, el IIBI (anteriormente CUIB) participó en las Reuniones de Expertos sobre un Código Internacional de Catalogación, auspiciadas por la IFLA y celebradas entre 2003 y 2007 en las distintas regiones del mundo con la finalidad de "promover el desarrollo de un código internacional de catalogación para la descripción y el acceso bibliográfico". La primera de esta serie de reuniones fue celebrada en Frankfurt en 2003 para Europa y los catalogadores angloamericanos; la segunda en Buenos Aires en 2004 para América Latina y el Caribe; la tercera en el Cairo, Egipto en 2005 para los hablantes de árabe del Oriente Medio; la cuarta en Seúl, Corea del Sur, para los países asiáticos; y la quinta en Pretoria, Sudáfrica, en 2007, para los países del África subsahariana (IFLA. Cataloging Section, 2016).

Previamente a la reunión celebrada en Buenos Aires en agosto de 2004 (http://www.ifla.org/node/10599), el entonces Centro Universitario de Investigaciones Bibliotecológicas de la UNAM convocó a los catalogadores mexicanos a la Primera Reunión Nacional sobre un Código Internacional de Catalogación, la cual tuvo lugar en junio de 2004:

Esta reunión tuvo como objetivos generales: la revisión y actualización del documento preliminar de 2003 sobre las Declaraciones de los Principios Internacionales de Catalogación elaborados en Frankfurt, Alemania. Así como, discutir los problemas de interpretación y aplicación de las Reglas de Catalogación Anglo-Americanas en las bibliotecas mexicanas, con la finalidad de considerar las posiciones de los catalogadores mexicanos y preparar el documento que el Grupo Mexicano de Catalogadores presentaría en la $2^{a}$ Reunión IFLA de Expertos sobre un Código Internacional de Catalogación, a realizarse en agosto de 2004 en Buenos Aires, Argentina (Rodríguez García, 2004)

En esta reunión emblemática participaron especialistas de las instituciones bibliotecarias de la UNAM y de otras del país, entre ellas: la Biblioteca Nacional de México; la Dirección General de Bibliotecas de la UNAM; el Centro Universitario de Investigaciones Bibliotecológicas; el Colegio de Bibliotecología de la UNAM; la Biblioteca de las Artes del Consejo Nacional de la Cultura y de las Artes; la Universidad de San Luis; la Dirección de Bibliotecas de la Secretaria de Educación del Distrito Federal. 
Esta Primera Reunión Nacional sobre un Código Internacional de Catalogación dio origen a la serie de Encuentros de Catalogación y Metadatos, los cuales han sido auspiciados y organizados por el Centro Universitario de Investigaciones Bibliotecológicas, el ahora Instituto de Investigaciones Bibliotecológicas y de la Información. Estos han sido celebrados en las siguientes fechas y las memorias correspondientes han sido publicadas en Acceso Abierto:

- Segundo Encuentro Nacional de Catalogación y Metadatos: en los umbrales de un nuevo código de catalogación (24 al 26 de Octubre de 2007) http://132.248.242.6/ publica/conmutarl.php?arch=1\&idx $=214$

- III Encuentro de Catalogación y Metadatos (29 al 31 de Octubre de 2008) http://132.248.242.6/ publica/conmutarl.php?arch=1\&idx $=226$

- IV Encuentro de Catalogación y Metadatos (4 al 6 de Noviembre de 2009) http://132.248.242.3/ publica/archivos/libros/iv encuentro catalogacion.pdf

- V Encuentro de Catalogación y Metadatos: 2010, Año de la Investigación en Catalogación. Organizado por el Centro Universitario de Investigaciones Bibliotecológicas de la UNAM y la Biblioteca Nacional de México (29 de Septiembre al 10 . de Octubre de 2010)

http://132.248.242.6/ publica/conmutarl.php?arch $=1 \& i d x=258$

- VI Encuentro de Catalogación y Metadatos (21 al 23 de Septiembre de 2011) http://eprints.rclis.org/22890/

- VII Encuentro de Catalogación y Metadatos (5 al 7 de Septiembre de 2012) http://eprints.rclis.org/28162/1/7 enc cat meta 2012.pdf

- VIII Encuentro de catalogación y Metadatos (2014)

Estos encuentros se han convertido en el foro nacional y regional por excelencia para la discusión académica e intercambio de experiencias sobre la teoría y práctica de la organización de la información y la catalogación, particularmente sobre la adopción y aplicación del nuevo estándar de catalogación, RDA (Resource Description and Access), tema que ha estado presente en todos los encuentros. Las memorias de estos encuentros incluyen numerosos trabajos que han abordado diversos aspectos relacionados con la aplicación de RDA en diferentes bibliotecas mexicanas y latinoamericanas. Otros temas que han sido abordados en estos encuentros son: el modelo FRBR; control de autoridades (nombres y materias), catalogación de recursos electrónicos, relación entre catalogación y metadatos; Dublin Core y otros sistemas de metadatos; tendencias de la catalogación y nuevas tecnologías; características y problemas de los catálogos, opciones tecnológicas para el desarrollo del catálogo, programas cooperativos de catalogación, formación de bibliotecólogos en la organización de la información y la catalogación

En estos encuentros se ha contado con la presencia de Bárbara Tillet y de Ana Cristán de la Biblioteca del Congreso de los Estados Unidos, quienes han presentado las siguientes conferencias: "RDA (Resource Description and Access): Status report on the new cataloguing code" (B. Tillet); "Resultados e impacto de la prueba de las bibliotecas nacionales de EE.UU. sobre las RDA" (A. Cristán); "Implementación de RDA en la Library of Congress: impacto de la recodificación en la base de datos de autoridades" (A Cristan). 
Adicionalmente, otros especialistas también han presentado importantes conferencias, y dentro de ellas pueden mencionarse: "Implications of RDA on cataloging practice and research", por Shawne D. Miksa, Department of Library and Information Sciences, College of Information, University of North Texas; "El papel de los catalogadores en el siglo XXI", por Elaine Sanchez, Cataloging \& Metadata Services, Texas State University-San Marcos; "Dataset relationships in the bibliographic universo", por Jane Greenberg, Metdata Research Center. University of North Carolina at Chapel Hill; "Las RDA (Resource Description and Access) y su impacto en la catalogación y los catálogos: necesidades de investigación y Perspectivas de las relaciones en RDA", por Filiberto Felipe Martínez Arellano, Centro Universitario de Investigaciones Bibliotecológicas.

En estos encuentros se ha contado con la participación como ponentes de especialistas en catalogación de la Biblioteca Nacional de México, de la Dirección General de Bibliotecas de la UNAM y del Centro Universitario de Investigaciones Bibliotecológicas, además de otros de numerosas instituciones mexicanas. Asimismo, es importante mencionar la participación de ponentes de la Biblioteca Nacional del Perú y de la Biblioteca Nacional de Colombia, así como de otras instituciones de Estados Unidos, Canadá, España y América Latina, de entre ellas pueden mencionarse las siguientes: University of Washington; Texas A\&M University, Kent State University; McGill University, Canada; Universidad Carlos III de Madrid; Universitat de Barcelona, España; Universidad de Salamanca; Universidad Nacional de Cuyo, Argentina; Universidad de Buenos Aires; Universidad de Antioquia, Colombia; Universidad de la Salle, Bogotá; Universidad Técnica Federico Santa María, Chile; Universidad de Puerto Rico; Instituto de Información Científica y Tecnológica, Cuba; Universidad de la Habana; Biblioteca Casa de las Américas de Cuba. Asimismo, también se ha contado con la participación de ponentes de las siguientes instituciones de Brasil: Universidade Federal de São Carlos, Brasil; Universidade Júlio de Mesquita Filho, São Paulo; Doutorando em Ciência da Informação, Universidade Estadual Paulista; Programa de Pós-Graduação em Ciência da Informação, Estadual Paulista; Universidade Tecnológica Federal do Paraná; Universidad de São Paulo, Escuela de Comunicaciones y Artes, São Paulo.

\section{b) Encuentros Internacionales de Catalogación}

Por otro lado, la 2a Reunión IFLA de Expertos sobre un Código Internacional de Catalogación, celebrada en agosto de 2004 en Buenos Aires, Argentina, dio lugar al surgimiento y celebración en Latinoamérica de la serie de Encuentros Internacionales de Catalogación, los cuales se han efectuado en los siguientes lugares y fechas:

- I Encuentro Internacional de Catalogadores "Nuevas tendencias en la normalización y sistematización de la información." Biblioteca Nacional del Perú, Octubre de 2005.

- II Encuentro Internacional de Catalogación [i. e. Catalogadores] "Tendencias en la teoría y práctica de la catalogación bibliográfica". Centro Universitario de Investigaciones Bibliotecológicas y Biblioteca Nacional de México, Septiembre de 2016.

- III Encuentro Internacional de Catalogadores "Tendencias actuales en la organización de la información". Biblioteca Nacional de Argentina, Noviembre de 2007.

- IV Encuentro Internacional de Catalogadores "La organización de la información frente a los cambios de las normas internacionales." Biblioteca Luis Ángel Arango y Biblioteca 
Nacional de Colombia, Octubre de 2008.

- V Encuentro Internacional de Catalogadores "Organización de la información en el contexto de las nuevas normas internacionales". Biblioteca Nacional Pedro Henríquez Ureña, República Dominicana, Octubre de 2009.

- VI Encuentro Internacional de Catalogadores "Aplicaciones tecnológicas y la nueva normativa en el procesamiento técnico de la información: una visión para el cambio". Universidad de Costa Rica, Escuela de Bibliotecología y Ciencias de la Información, Octubre de 2010.

- VII Encuentro Internacional de Catalogadores "Estándares y procedimientos para la organización de la información". Biblioteca Nacional de Argentina, Noviembre de 2011.

- VIII Encuentro Internacional de Catalogadores "Perspectivas sociales de la catalogación". Biblioteca Nacional de la República Bolivariana de Venezuela, Noviembre 2012.

- IX Encontro Internacional de Catalogadores "Catalogação: do real ao virtual". Fundação Biblioteca Nacional do Brasil, Octubre 2013.

- X Encuentro Internacional de Catalogadores "Normalización técnica, tecnológica y legislativa de la catalogación". Archivo y Biblioteca Nacionales de Bolivia, Septiembre 2015.

Como se ha mencionado anteriormente, el II Encuentro Internacional de Catalogadores fue organizado por el Centro Universitario de Investigaciones Bibliotecológicas y la Biblioteca Nacional de México, y fueron publicadas las memorias del mismo en Acceso Abierto. (http://132.248.242.6/ publica/conmutarl.php?arch=1\&idx=191).

Los objetivos de este evento académico fueron:

- Compartir e intercambiar experiencias sobre la teoría y la práctica catalográfica en las bibliotecas de América Latina y de otros países del extranjero.

- Discutir las tendencias de la catalogación en el marco de las nuevas tecnologías de información y a la luz de la revisión de los Principios Internacionales de Catalogación.

- Fomentar la participación en programas de cooperación de catalogación y de control de autoridades en el ámbito internacional.

- Delinear pautas, recursos y procedimientos para la catalogación y el control de las autoridades en forma cooperativa.

- Discutir el perfil para la formación del bibliotecario en el área de la catalogación y de la organización de la información.

Entre los temas que se abordaron en este encuentro se pueden mencionar los siguientes:

- Tendencias en los principios internacionales de catalogación

- Requisitos Funcionales de los Registros Bibliográficos (FRBR) 
- De las AACR2 hacia las RDA

- Catalogación de publicaciones seriadas

- Catalogación de recursos continuos

- Catalogación de recursos electrónicos

- Control de autoridades de nombres y de materias

- Metadatos y catalogación

- Cooperación en catalogación y autoridades

Asimismo, en este evento se contó con la participación de la Dra. Barbara Tillet de la Biblioteca del Congreso de los E. U., quien impartió la conferencia "RDA y la influencia de FRBR y otras iniciativas de IFLA", la de Ana Cristán de la misma institución, con la conferencia "Los programas de cooperación en catalogación de Library of Congress de EUA (LC) en el ámbito internacional: ¿éxitos o fracasos?", y la de Ageo García Barbabosa de la Universidad de Tulane, quien presentó la ponencia "La normalización internacional y el control de autoridades en las redes regionales de catalogación cooperativa: la experiencia latinoamericana". Además de la participación de los profesionales de la Biblioteca Nacional de México y del Centro Universitario de Investigaciones Bibliotecológicas, así como de otras instituciones mexicanas, se contó con ponentes de Argentina, Brasil, Colombia, Cuba, Guatemala, Perú y Estados Unidos e Italia, que representaron a las siguientes instituciones: Universidad de Buenos Aires, Argentina; Universidad de Brasilia; Universidade Estadual de Maringá, Brasil; Biblioteca Luis Ángel Arango, Colombia; Universidad Francisco Marroquín, Guatemala; Biblioteca Nacional del Perú; Universidad Nacional Mayor de San Marcos, Perú; Pontificia Universidad Católica del Perú; Kent State University, Association for Library Collections and Technical Services, Cataloging and Clasification Section; Cataloging \& Classification Quartely; Università degli Studi di Udine; Università degli Studi di Firenze.

\section{c) Seminario de Organización de la Información}

Por otro lado, es conveniente hacer mención que el IIBI ha auspiciado el Seminario de Organización de la Información (http://iibi.unam.mx/seminarios.html), cuyos objetivos son:

- Analizar la eficiencia y efectividad del proceso de catalogación a partir de la adopción del nuevo código de descripción y acceso a recursos.

- Evaluar los efectos de utilizar modelos conceptuales en los catálogos en línea, sus interfaces, recursos disponibles y estructuras de metadatos.

- Promover la participación en proyectos de investigación en catalogación a nivel local, nacional y regional.

- Compartir las experiencias nacionales e internacionales con respecto a la necesidad de llevar a cabo el Control Bibliográfico, la catalogación cooperativa y el uso del catálogo.

- Discutir el impacto actual de la investigación en catalogación en los planes y programas de formación de bibliotecólogos en el área de organización de la información. 
En este seminario se han efectuado reuniones periódicas de discusión sobre asuntos de la organización de la información y la catalogación, en las que participaron, además de los profesionales de la Biblioteca Nacional de México, de la Dirección General de Bibliotecas de la UNAM y del Instituto de Investigaciones Bibliotecológicas y de la Información, los profesores del área de catalogación de las dos escuelas de Bibliotecología de la Ciudad de México: El Colegio de Bibliotecología de la UNAM y la Escuela Nacional de Bibliotecología y Archivonomía. Asimismo, es importante señalar la participación de los alumnos de la Maestría y del Doctorado en Bibliotecología y Estudios de la Información de la UNAM, quienes se encuentran desarrollando tesis sobre organización de la información y la catalogación. Adicionalmente, han participado en este seminario profesionales de América Latina y de España.

\section{Conclusiones}

Indudablemente, la existencia dentro de la Universidad Nacional Autónoma de México de tres instituciones bibliotecarias con objetivos estrechamente relacionados con la organización de la información y la catalogación hacen de esta institución un espacio sin igual donde convergen distintas perspectivas sobre este tipo de asuntos. En relación con la aplicación e implementación de las RDA se han conjugados los aspectos teóricos con los aspectos prácticos de este asunto. El IIBI se ha encargado del análisis teórico de la implementación de las RDA, mientras que la Biblioteca Nacional de México se abocó a su aplicación en una institución que compila los materiales publicados en este país y la Dirección General de Bibliotecas de los libros y otro tipo de materiales adquiridos para apoyar las actividades de investigación y docencia de la UNAM, acervos totalmente distintos entre estas dos instituciones, los cuales presentan características y retos diferentes. La sinergia entre estas tres instituciones ha fructificado en una comprensión amplia y global del nuevo estándar de catalogación y de los retos que implica su implementación en diferentes ambientes bibliotecarios. Por otro lado, la integración de las escuelas de bibliotecología de licenciatura y del Posgrado en Bibliotecología y Estudios de la Información en el análisis de la problemática de la aplicación de la RDA, reditúa en una mejor formación de los cuadros profesionales del área de catalogación. Esta situación particular de la UNAM, en la que convergen tres importantes instituciones bibliotecarias del país, ha hecho que la misma se haya convertido en una institución con capacidad de convocatoria para el análisis y la discusión de los retos que implican el conocimiento y aplicación de las RDA, tanto a nivel nacional como regional, lo cual ha generado una serie publicaciones como resultado de dicho análisis y discusión.

Agradecimiento:

Se agradece al Departamento de Procesos Técnicos y al Departamento de Publicaciones Periódicas y Mapas de la Dirección General de Bibliotecas de la UNAM su apoyo en la elaboración de la parte correspondiente a esta institución

\section{Referencias bibliográficas}

García López, C., Mejía Ruiz, J. A., Ramos Díaz, M. de los A. y Guzmán Rodríguez, E. M. (2012). LIBRUNAM: actualizado y renovado (2012). México: UNAM, Dirección General de Bibliotecas. 
Granados Xolalpa, E. y Mendoza Benítez, L. (2014). Manual de información básica en bibliotecología. México: UNAM, Dirección General de Bibliotecas.

IFLA. Cataloging Section (2016). IFLA Meetings of Experts on an International Cataloguing Code. Recuperado de http://www.ifla.org/node/576

Martínez Arellano, F. F. (2007). XXV años del Centro Universitario de Investigaciones Bibliotecológicas: testimonios: Presentación. México: UNAM. Centro Universitario de Investigaciones Bibliotecológicas. Recuperado de: http://132.248.242.6/ publica/conmutarl.php?arch=1\&idx=190

Rodríguez García, A. A. (2004). Primera Reunión Nacional sobre un Código Internacional de Catalogación 24 y 25 de Junio de 2004, Ciudad de México, D, F. Biblioteca Universitaria, Nueva Época, 2, 185-190. Recuperado de http://www.redalyc.org/html/285/28570211/

Rodríguez García, A. A. (2012). Curso: Las complejidades para la implementación de los lineamientos RDA. (Documento no publicado).

UNAM (2015). Memoria UNAM 2015: Dirección General de Bibliotecas Recuperado de http://www.planeacion.unam.mx/Memoria/2015/PDF/14.2-DGB.pdf

UNAM (2016). La UNAM en números 2015-2016. Recuperado de http://www.estadistica.unam.mx/numeralia/

UNAM. Biblioteca Nacional de México (2016). Misión, visión y objetivos. [Sitio web]. Recuperado de http://bnm.unam.mx/index.php/mision-vision-y-objetivos

UNAM. Dirección General de Bibliotecas (2013). Sistema Bibliotecario y de Información. Recuperado de http://www.dgbiblio.unam.mx/index.php/acerca-del-sistema

UNAM. Instituto de Investigaciones Bibliotecológicas y de la Información (2017). Proyectos de Investigación. Recuperado de http://iibi.unam.mx/proyindividual.html 